$$
\text { CONF- } 960334--1
$$

UCRL-JC-123448

PREPRINT

\title{
FABRICATION AND TESTING OF THERMOELECTRIC THIN FILM DEVICES
}
Andrew V. Wagner
Ronald J. Foreman
Leslie J. Summers
Troy.W. Barbee, Jr.
AFR is 2 iss
OSTI

Fen

This paper was prepared for submittal to the

ICT96 XV International Conference on Thermoelectrics,

Pasadena, California, March 25, 1996

March 1996

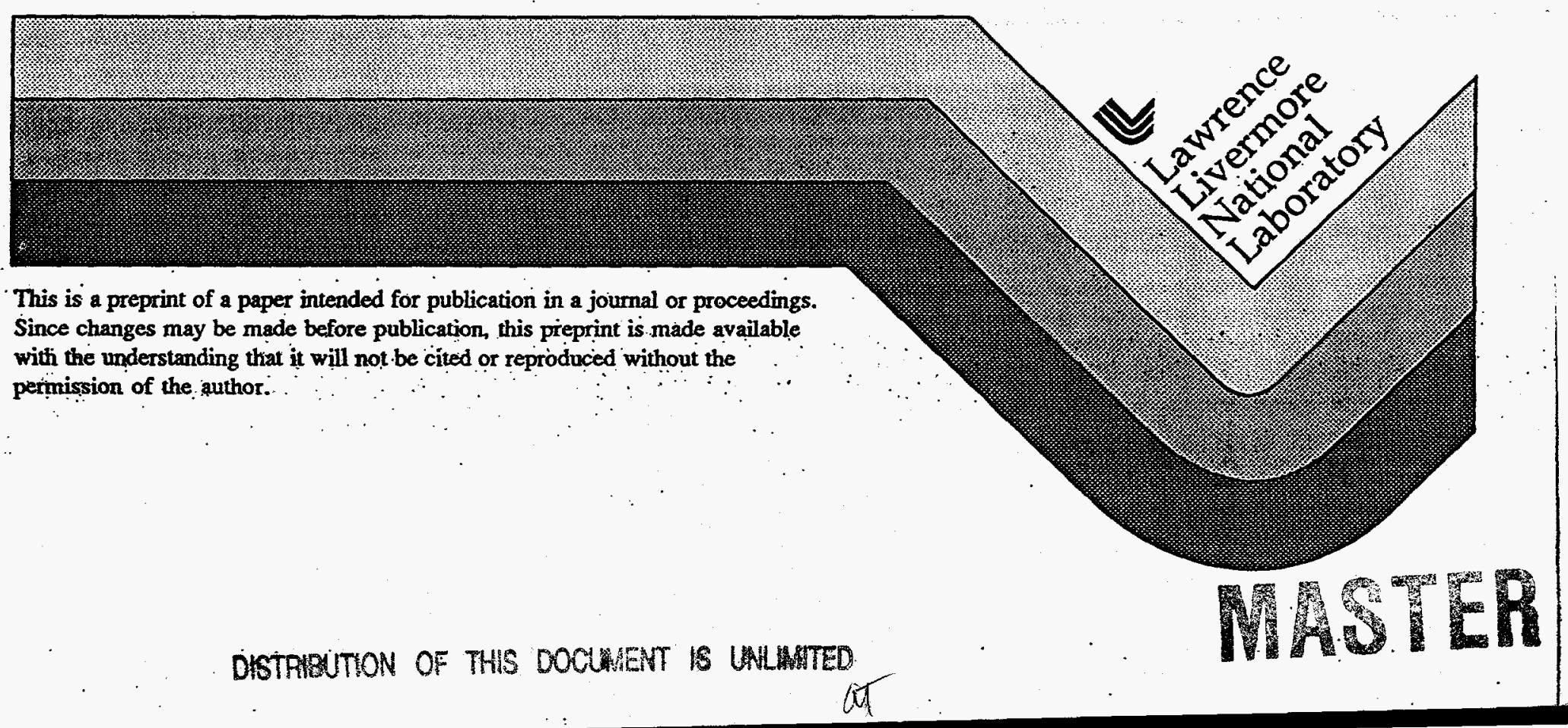




\section{DISCLAMMER}

Portions of this document may be illegible in electronic image products. Images are produced from the best available original document. 


\title{
FABRICATION AND TESTING OF THERMOELECTRIC THIN FILM DEVICES
}

\author{
Andrew V. Wagner, Ronald J. Foreman, Leslie J. Summers, \\ Troy W. Barbee Jr., and Joseph C. Farmer \\ Chemistry and Materials Science Department \\ Lawrence Livermore National Laboratory \\ 7000 East Avenue Livermore, CA 94550 USA
}

\begin{abstract}
Two thin-film thermoelectric devices are experimentally demonstrated. The relevant thermal loads on the cold junction of these devices are determined. The analytical form of the equation that describes the thermal loading of the device enables us to model the performance based on the independently measured electronic properties of the films forming the devices. This model elucidates which parameters determine device performance, and how they can be used to maximize performance.
\end{abstract}

\section{Introduction}

Thermoelectric materials play an important technological role as sensors, power generators and solid-state coolers. The use of bulk thermoelectric material for cooling is becoming more widespread. For example portable thermoelectric food coolers are now becoming common place. Thermoelectrics have also found a niche where vibration free cooling is critical such as in infrared detectors and low-light $\mathrm{CCD}$ array cameras. They are also used as a convenient way of quickly heating and cooling for DNA synthesis by thermal cycling. These applications all make use of bulk thermoelectric materials, but there is increasing interest in thin-film thermoelectric devices.

Here we focus on the use of thermoelectrics for cooling, which is the most demanding application for such thinfilm materials. Thin-film coolers could be incorporated directly into semiconductor devices in close proximity to regions such as the sensing element in IR detectors. This could greatly reduce the amount of material being cooled, thereby reducing the energy required for cooling. Low energy consumption is critical in building hand-held, battery-operated devices. A thin-film deposition technology would also be compatible with the planar technology essential to the construction of integrated circuits. Further interest in thin film-coolers stems from potential improvement in thermoelectric figure of merit from two dimensional confinement of the charge carriers in quantum well structures. [I]

The problem of thin film deposition of thermoelectrics has been worked on sporadically since the 1950's. Numerous deposition techniques have been tried including evaporation, flash evaporation, MBE, CVD, sputtering, and laser ablation. In the case of compound semiconductors, a primary difficulty is maintaining the stoichiometry of the growing film. Keeping sufficient purity of material and controlling the dopant levels are also critical. The growth structure is another important issue since grain boundaries and crystalline defects effect the resistivity of the deposited film by scattering charge carriers.

Recent thin-film device work has begun to show promise of producing useful devices. ${ }^{[2]}$ Much of the experimental work involves optimizing the growth parameters to achieve the best quality films.

In this work we investigate which heat transport factors are essential to consider when working with thin-film thermoelectric devices. If the heat transport to the cold junction from all sources can be calculated then the electrical conductivity and Seebeck coefficient can be used to predict the cooling achievable by the device. Here we calculated device performance based on the independently-measured thermoelectric properties of the individual films comprising a device. We compare this with experimental measurements of the maximum cooling of operating devices. The model developed and discussed here can be used as a means for optimizing device performance.

\section{Experimental techniques}

Eilm growth and optimization:

Magnetron sputtering was chosen for the de position of thin film devices for two reasons. First, sputtering allows multicomponent materials to be deposited from a single source without many of the compositional shifts associated with thermal evaporation techniques. Second, the technology for large-scale deposition has been developed in industry and has been shown capable of producing large quantities of material necessary for commercially viable thin film thermoelectric devices. We discuss the sputtering process used to deposit these films in detail elsewhere. ${ }^{[3]}$

Film quality is extremely important in growing devices. Degradation of the electrical properties of the films are reflected in reduced cooling capacity of the device. The individual $\mathbf{p}$ and $n$-type films were optimized independently. The electrical resistivity and Seebeck coefficient of the individual films was measured independently. The structural quality was investigated using $\mathrm{X}$-ray diffraction, which allows the degree of orientation to be investigated, as well as the crystalline structure. Scanning electron microscopy (SEM) was used to measure the film thickness as well as characterize growth structure. The results of these measurements were used 
as the basis for directed changes in the sputter target composition as well as deposition parameters.

For the $\mathrm{Bi}_{0.9} \mathrm{Sb}_{0.1}$ film used as the n-type leg of one of the devices discussed below, the main issues were crystal quality, crystal size, and impurities. By raising the deposition temperature the electronic properties of the films were improved as a result of increased crystal size and quality. Increasing substrate deposition temperature was limited by island formation at higher temperatures. The pressure of the sputtering gas and sample bias were also found to effect the properties of the film. ${ }^{[4]}$ The $\mathrm{Bi}_{0.9} \mathrm{Sb}_{0.1}$ film used in the device discussed here was deposited at approximately $180^{\circ} \mathrm{C}$.

Similar observations were made for the $\left(\mathrm{Bi}_{1-x} \mathrm{Sb}_{x}\right)_{2}\left(\mathrm{Te}_{1-y} \mathrm{Se}_{y}\right)_{3}$ films. The problem of island formation could be reduced by beginning deposition at a lower temperature and quickly raising the temperature during deposition. The difference in vapor pressure of the components makes deposition at these high temperatures difficult. However we have shown that by using a "hot walled" environment and excess Te concentration in the sputter target the stoichiometry of the resulting films could be controlled. ${ }^{[3]}$

Mica was chosen as the substrate material since mica can be easily cleaved into extremely thin sheets. It is important that the substrate be thin in order to minimize thermal conductivity losses in the substrate. In addition mica has a hexagonal symmetry which is the same as the film, the cleaved surfaces are flat, and it is able to withstand the high temperature necessary for high quality deposition. The "hot walled" environment needed to control the stoichiometry also helps maintain the thin mica substrates at the desired temperature. This is particularly important since the flexible mica substrates essential to making devices do not make good mechanical contact with the stage and there is little lateral heat transport in the mica from the points where it does contact the stage. Thus, without a "hot walled" environment the temperature of the substrates would be in radiative equilibrium somewhere between the stage temperature and the chamber temperature.

The devices were prepared by first depositing one leg of the device through a mask onto the mica substrate. The chamber is then vented, the mask reset to give a small junction, and the second film deposited.

Care must be taken in depositing the second leg of the device. The existing film changes the thermal characteristics of the mica substrate and may lead to increased substrate temperature near the junction during deposition. The second film does not nucleate growth at the edge of the first deposition. A small gap is formed by atoms deposited near the junction migrating to the edge of the existing film instead of nucleating the growth of the new film. If such a small gap forms at the junction the performance of the device is destroyed. To prevent this problem, lower temperatures are used at the beginning of the deposition.

Measurement technique:

To test the device it was bridged (face down) between two copper blocks and attached with conductive silver paint

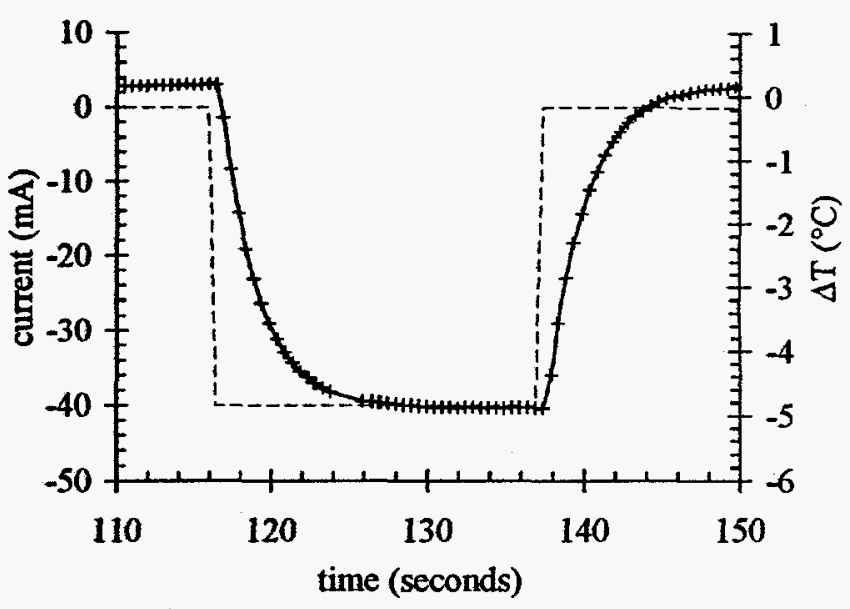

Figure 1: Measured temperature response for device 1 to a $40 \mathrm{~mA}$ current pulse. The zero offset for the cooling for zero current is a result of slight nonequlibrium of the heat sink with the surrounding radiative environment. These small offsets show the importance of the pulsed technique.

which provides both electrical and thermal contact. One thermocouple is attached to the back side of the mica substrate at the cold junction. A second thermocouple monitors the temperature of one of the copper contact blocks. Both thermocouples are electrically isolated from the current pulse used to produce cooling in the device by the mica substrate material. The device is tested by sending a current pulse to the device and comparing the temperature difference between the two thermocouples before and during the pulse. The current pulses sent to the device are controlled by the data acquisition computer and can be varied in amplitude and duration. The computer allows the pulse duration to be set so that the temperature difference can be compared after the cooling transient which is typically less than a couple seconds (figure 1). Furthermore, the pulse current is varied to explore the response of the device as a function of current. It is important that the entire chamber be in thermal equilibrium with the copper blocks that are used as the "hot side" of the device. This is necessary in order for the equations governing radiative losses discussed below to apply.

Since the chosen substrate is a very good insulator, our standard measurement techniques could be used to measure the electronic properties of each leg of the device independently ${ }^{[s]}$. This enables the independent calculation of expected device performance for comparison with measured results.

\section{Calculations}

In building thermoelectric cooling devices the relative importance of the various heat loads on the cold junction must be understood. To optimize device performance, it is useful to develop a comprehensive analytical model.

Thin-film thermoelectric devices which are designed to transport heat in the plane of the film are inherently low-power devices. Thus, small secondary heat loadings of the cold junction, which are usually inconsequential for bulk devices, are very important to consider for thin-film devices. For a device made of bulk material, thermal conductivity in the 


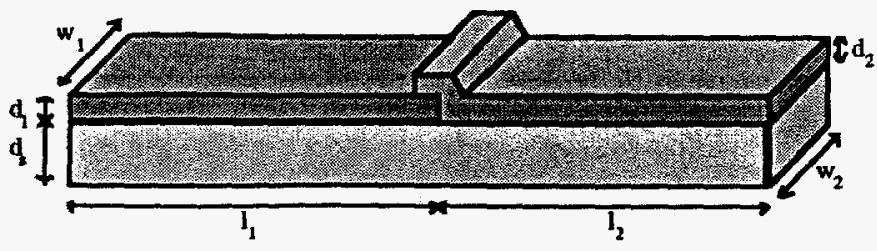

Figure 2: Schematic of a simple cooler device showing a p and n-type film on a thin substrate.

thermoelectric material is the dominant source of heat transfer to the cold junction and thus limits the minimum temperature to which the device can cool. In thin films however, other losses are equally important. These losses include thermal conduction in the substrate supporting the film, coupling to the environment by radiation, and conduction and convection in the atmosphere. Losses in the atmosphere were neglected in the discussion below since our experiments were conducted in vacuum. The load being cooled must also be considered, including the thermocouple used to monitor the device.

Analytical expressions for the thermal loads are developed for the simple geometry where the p-n junction is deposited on one side of a cleaved mica substrate as shown in figure 2. The load from each side of the device and each leg of the measurement thermocouple will be considered separately.

The contribution of thermal conductivity in the film and substrate to the heat transfer coefficient of the device can be calculated as follows:

$$
\begin{aligned}
& K_{f i l m}=\kappa_{f i l m} \frac{d w}{l} \\
& K_{s u b}=K_{s u b} \frac{d_{s} w}{l}
\end{aligned}
$$

As the surface of the device gets larger, radiation plays an increasingly important role. For small temperature differences, the coefficient of heat transfer for a surface $(\mathrm{H})$ is the derivative of the radiance $(\Re)$ :

$$
\Re=e \sigma T^{4}
$$

where $\sigma$ is the Stefan-Boltzmann constant $\left(5.67 \times 10^{-12} \mathrm{~W} / \mathrm{cm}^{2} \mathrm{~K}^{4}\right)$ and $e$ is the emissivity. The heat transfer coefficient across the surface at the reference temperature $\left(T_{0}\right)$ is:

$$
H=\left.\frac{\partial R}{\partial T}\right|_{T_{o}}=4 e \sigma T_{o}^{3}
$$

Heat will be lost from both the top and bottom surfaces in an actual device. However, this factor of two will be offset by the fact that the average temperature of the device will be halfway between the temperature of the cold junction and the reference temperature. It is assumed that the whole radiative environment is at the reference temperature and therefore the radiative heat transfer coefficient for the device is:

$$
K_{\text {rad }}=H l w
$$

Heat losses in the measurement thermocouples must also be estimated. Despite the use of extremely thin thermocouples (much thinner than a hair), they are still several times thicker than the film that is being tested. Furthermore, metallic thermocouples have a much higher thermal conductivity than any of the other components in the system. Although relatively long thermocouple wires are used to minimize thermal losses from conduction, the heat transferred down the thermocouple is radiated away at the surface of the thermocouple. In our case the loss due to the thermocouples leads can be approximated by the analytical solution for the heat loss by radiation from a semi-infinite rod. ${ }^{[6]}$ The temperature distribution in the rod is:

$$
T=T_{o}+\left(T_{c}-T_{o}\right) e^{-x \sqrt{p H / \omega K_{T_{c}}}}
$$

where $p$ the perimeter, $2 \pi r$, $\omega$ is the cross-sectional area, $\pi r^{2}$; and $r$ is the radius of the wire. The rate of heat flow down the wire is:

$$
\begin{aligned}
\left.\omega \kappa_{T c} \frac{\partial T}{\partial x}\right|_{x=0} & =\pi r^{2} \kappa_{T c}\left(T_{c}-T_{0}\right) \sqrt{\frac{2 H}{r \kappa_{T c}}} \\
& =\left(T_{c}-T_{0}\right) \pi r^{3 / 2} \sqrt{2 H \kappa_{T c}}
\end{aligned}
$$

The heat flow coefficient for each leg of the thermocouple is:

$$
K_{T c}=\pi r^{3 / 2} \sqrt{2 H \kappa_{T c}}
$$

To validate the assumption of the semi-infinite rod geometry, the length of thermocouple at which the temperature will be half-way between the cold junction and the reference temperature can be calculated as follows from equation 6:

$$
\begin{gathered}
\frac{1}{2}=\frac{T-T_{o}}{\left(T_{c}-T_{o}\right)}=e^{-x \sqrt{2 H / r \kappa_{T c}}} \\
x^{*}=-\ln \left(\frac{1}{2}\right) \sqrt{\frac{1}{2} r \frac{\kappa_{T c}}{H}}
\end{gathered}
$$

For the 0.001 inch diameter thermocouples used during this work, $\mathrm{x}^{*}$ is approximately half a centimeter. This is small compared to the length of the thermocouples used in the experiments. The length of thermocouple needed to remain in this approximation varies as the square root of the thermocouple radius.

The performance of a device is calculated with the figure of merit $(Z)$ of the device. To calculate the performance of an actual device, one must sum over all the thermal losses and resistances in the system. In the simple device sketched in figure 2 several assumptions were made. First, it was assumed that the overlap of the two layers is small compared to other dimensions and can be ignored. Similarly, it was assumed that there is no junction resistance and no interdiffusion or intermixing of the two layers at the junction which could degrade the thermoelectric properties of the materials in the vicinity of the junction. Finally, the thickness of the film and substrate is small enough compared to the lateral dimensions that radiative losses from the edges can be ignored.

The Seebeck coefficient $(\alpha)$, resistance $(R)$ and heat transfer coefficients for the device as a whole $(\mathrm{K})$ can then be calculated, and from these the figure of merit of the device can be calculated:

$$
\alpha=\alpha_{1}-\alpha_{2}
$$




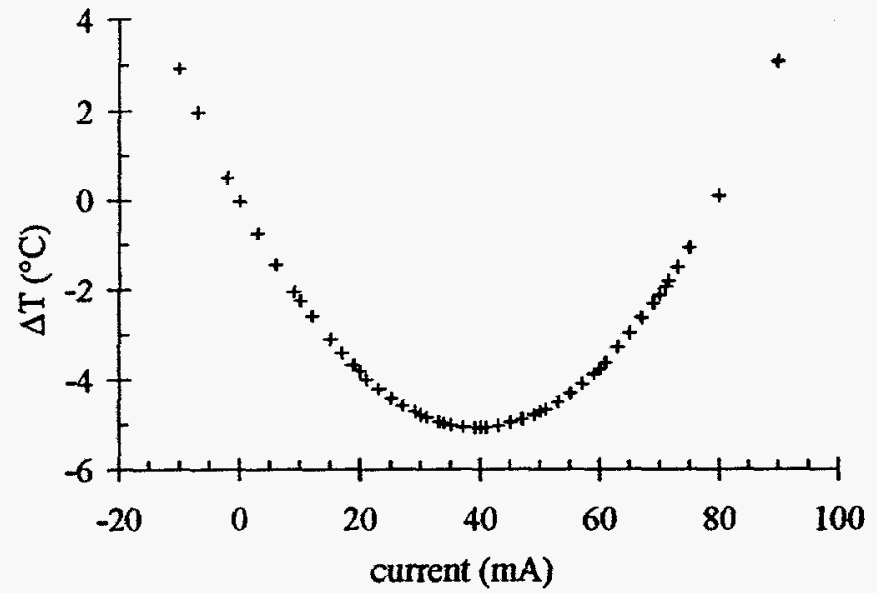

Figure 3: Measured cooling for device 1 operated at room temperature.

$$
\begin{gathered}
R=\frac{\rho_{1} l_{1}}{d_{1} w_{1}}+\frac{\rho_{2} l_{2}}{d_{2} w_{2}} \\
K=\sum_{p, n} K_{f i l m}+K_{s u b}+K_{r a d}+K_{T c} \\
Z=\frac{\alpha^{2}}{K R}
\end{gathered}
$$

From $\mathrm{Z}$ the maximum cooling achievable can be calculated:

$$
\Delta T_{\max }=T_{H}+\frac{1}{Z}\left(1-\sqrt{1+2 Z T_{H}}\right)
$$

\section{Results and discussion}

Two thin-film devices based on different materials will be discussed. The first device is a junction between a p-type $\left(\mathrm{Bi}_{0.25} \mathrm{Sb}_{0.75}\right)_{2}\left(\mathrm{Te}_{0.95} \mathrm{Se}_{0.05}\right)_{3}$ and an n-type $\mathrm{Bi}_{0.9} \mathrm{Sb}_{0.1}$ film on a $25 \mu \mathrm{m}$ thick mica substrate. The second device is on a $10 \mu \mathrm{m}$ thick mica substrate and uses a similar p-type material $\left(\mathrm{Bi}_{0.25} \mathrm{Sb}_{0.75}\right)_{2} \mathrm{Te}_{3}$ and with an n-type $\left(\mathrm{Bi}_{0.95} \mathrm{Sb}_{0.05}\right)_{2}\left(\mathrm{Te}_{0.95} \mathrm{Se}_{0.05}\right)_{3}$. The masks used in the deposition were arranged so that the films overlap slightly to form the cold junction of the device (as sketched in figure 2). Small devices (and their mica substrate) are then cut from the original film. Care must be taken while cutting the films to minimize damage to the thermoelectric material. In practice this damage and difficulty mounting the samples limit the minimum device size.

The electronic properties of the individual legs were measured independently on a mica witness substrate placed next to the device substrate for the deposition. The electronic properties of the witness sample and thickness were measured independently. The lateral dimensions of the device were also measured after it was mounted in the measurement jig. These measured parameters are recorded in table 1 and were used to calculate expected values for device performance.

The cooling of the actual devices was measured experimentally as a function of current (figure 3,4). The temperature response to a current pulse is also shown (figure 1). Much of the time required for response comes from the energy needed to heat the tiny dot of silver paint used to hold the thermocouple to the back of the mica substrate.

\begin{tabular}{|c|c|c|c|c|c|}
\hline & & device 1 & & device 2 & \\
\hline & & n-type & p-type & n-type & p-type \\
\hline & units & $\mathrm{Bi}_{0}, 5 \mathrm{Sb}_{0,1}$ & $(\mathrm{Bi}, \mathrm{Sb})_{2}(\mathrm{Te}, \mathrm{Se})_{3}$ & $(\mathrm{Bi}, \mathrm{Sb})_{2}(\mathrm{Te}, \mathrm{Se})_{3}$ & $\left(\mathrm{Bi}, \mathrm{Sb}_{2} \mathrm{Te}_{3}\right.$ \\
\hline & & \multicolumn{2}{|c|}{ measured values } & & \\
\hline$\alpha$ & V/K & $-1.00 \mathrm{E}-04$ & $1.10 \mathrm{E}-04$ & $-8.50 \mathrm{E}-05$ & $1.20 \mathrm{E}-04$ \\
\hline$\rho$ & $\Omega \mathrm{cm}$ & $5.70 \mathrm{E}-04$ & $1.00 \mathrm{E}-03$ & $9.40 \mathrm{E}-04$ & $6.20 \mathrm{E}-04$ \\
\hline 1 & $\mathrm{~cm}$ & 0.25 & 0.18 & 0.28 & 0.28 \\
\hline $\mathbf{w}$ & $\mathrm{cm}$ & 0.20 & 0.22 & 0.35 & 0.35 \\
\hline d & $\mathbf{c m}$ & $9.50 \mathrm{E}-04$ & $6.00 \mathrm{E}-04$ & $9.40 \mathrm{E}-04$ & $6.20 \mathrm{E}-04$ \\
\hline$d_{\text {mica }}$ & $\mathrm{cm}$ & $2.50 \mathrm{E}-03$ & & $1.03 \mathrm{E}-03$ & \\
\hline \multirow[t]{2}{*}{$\underline{\mathbf{I}_{\mathrm{T}}}$} & $\mathbf{c m}$ & $1.30 \mathrm{E}-03$ & $1.30 \mathrm{E}-03$ & $1.30 \mathrm{E}-03$ & $1.30 \mathrm{E}-03$ \\
\hline & & \multicolumn{2}{|c|}{ assumed values } & & \\
\hline$K_{\text {film }}$ & $\mathrm{W} / \mathrm{cm}^{2} \mathrm{~K}$ & \begin{tabular}{|l|}
0.080 \\
\end{tabular} & 0.014 & 0.014 & 0.014 \\
\hline$K_{\text {mica }}$ & $\mathrm{W} / \mathrm{cm}^{2} \mathrm{~K}$ & 0.020 & & & \\
\hline$K_{T c}$ & $\mathrm{~W} / \mathrm{cm}^{2} \mathrm{~K}$ & 0.300 & 0.190 & 0.300 & 0.190 \\
\hline$e_{\text {fis }}$ & & 0.600 & & & \\
\hline \multirow[t]{2}{*}{$e_{r_{c}}$} & & 0.300 & & & \\
\hline & & \multicolumn{2}{|c|}{ calculated values } & & \\
\hline$Z_{\text {fim }} T$ & & \begin{tabular}{|l|}
0.07 \\
\end{tabular} & 0.26 & 0.16 & 0.50 \\
\hline $\mathbf{K}_{\text {film }}$ & $\mathrm{W} / \mathrm{K}$ & $6.08 \mathrm{E}-05$ & $1.03 \mathrm{E}-05$ & $1.65 \mathrm{E}-05$ & $1.09 \mathrm{E}-05$ \\
\hline $\mathrm{K}_{\text {gub. }}$ & $\mathrm{w} / \mathrm{K}$ & $4.00 \mathrm{E}-05$ & $6.11 \mathrm{E}-05$ & $2.58 \mathrm{E}-05$ & $2.58 \mathrm{E}-05$ \\
\hline $\mathbf{K}_{\text {rad }}$ & W/K & $1.84 \mathrm{E}-05$ & $1.45 E-05$ & $3.60 \mathrm{E}-05$ & $3.60 \mathrm{E}-05$ \\
\hline $\mathbf{K}_{\mathbf{T \varepsilon}}$ & $\mathrm{W} / \mathrm{K}$ & $1.55 \mathrm{E}-06$ & $1.23 \mathrm{E}-06$ & $1.55 \mathrm{E}-06$ & $1.23 \mathrm{E}-06$ \\
\hline \multirow[t]{2}{*}{$\mathbf{R}$} & $\Omega$ & 0.75 & 1.36 & 0.80 & 0.80 \\
\hline & & \multicolumn{3}{|c|}{ whole device calculated values } & \\
\hline $\mathbf{K}$ & $\mathrm{w} / \mathrm{k}$ & $2.08 \mathrm{E}-04$ & & $1.54 \mathrm{E}-04$ & \\
\hline$\alpha$ & $\mathrm{V} / \mathrm{K}$ & $2.10 \mathrm{E}-04$ & & $2.05 \mathrm{E}-04$ & \\
\hline$\underline{\mathbf{R}}$ & $\Omega$ & 2.11 & & 1.60 & \\
\hline ZT & & 0.03 & & 0.05 & \\
\hline \multirow[t]{2}{*}{$\Delta T_{\max }$} & $\mathrm{K}$ & 4.39 & & 7.32 & \\
\hline & & \multicolumn{3}{|c|}{ measured device properties } & \\
\hline$\Delta T_{\max }$ & $\mathrm{K}$ & 5.10 & & 5.30 & \\
\hline
\end{tabular}

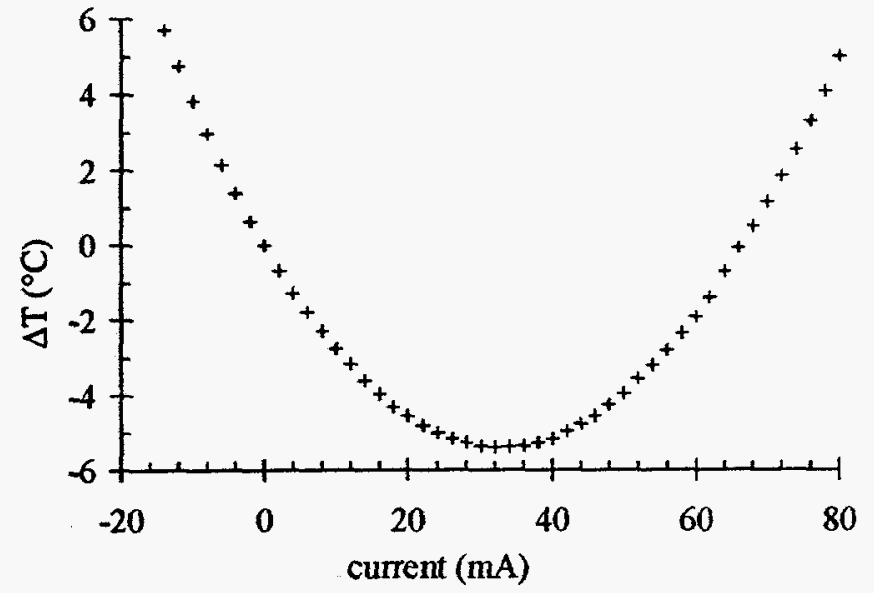

Figure 4: Measured cooling for device 2 operated at room temperature.

Table 1: At the top are the measured properties of the individual films used in the devices. Below are assumed values for the thermal conductivity and emissivity. The next section shows the heat transfer coefficients with the relative losses in each component. Finally, calculated values for $\Delta T_{\max }$ from the measured film properties can be compared with measured values for the devices listed at the bottom of the table. 


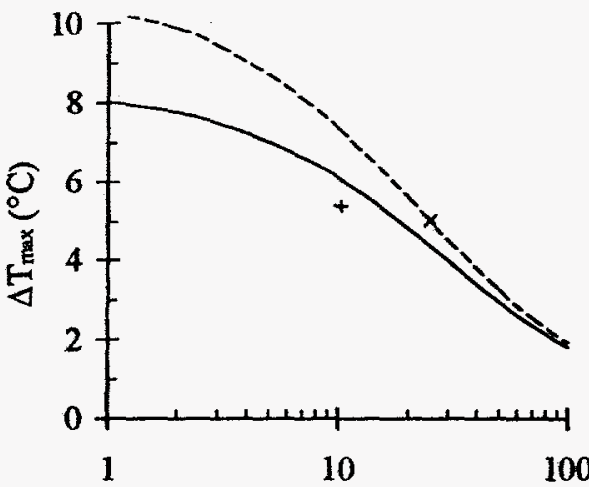

a) substrate thickness $(\mu \mathrm{m})$

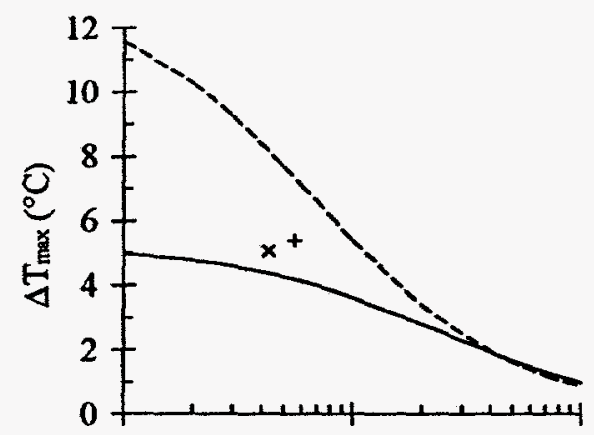

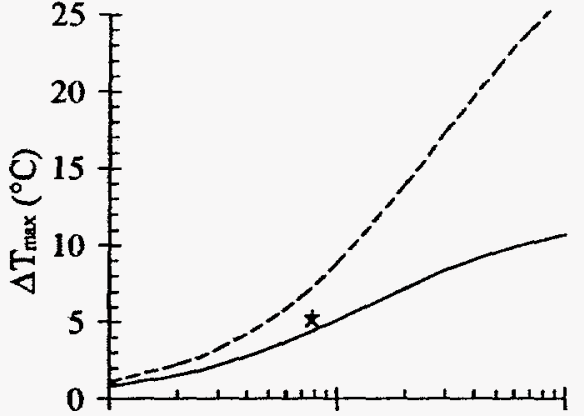

100
1

10 b)
10

1

device length (mm)

c)

average film thickness $(\mu \mathrm{m})$

Figure 5: Derived dependence of the maximum cooling with: a) substrate thickness; b) device length; $c$ ) average film thickness. The measured electronic properties and dimensions of the films used in the two devices were used in the calculations. In $b$ and $c$ the device leg length or film thickness rations are preserved. In both cases optimization in these ratios, which could be obtained from the model, could lead to further enhanced performance. Calculations based on device 1 are plotted as a solid line and device 2 as a dashed line. Measured values for device performance are also shown: $(x)$ device $1 ;(+)$ device 2 .

The cooling as a function of current (I) can also be calculated from the measured device parameters and compared with measured values:

$$
\Delta T=\frac{\alpha I T_{H}-\frac{1}{2} I^{2} R}{(\alpha I+K)}
$$

This is approximately parabolic as seen in figures 3 and 4 if $\mathrm{K} \gg>\alpha$, but switches to a more linear behavior at large $I$.

It is valuable to look more closely at the calculated values of the thermal loading for each device shown in table 1 to learn which loads are the largest, and thus what route would improve device performance the most. Table 1 shows that the thermal losses in the substrate are substantial. Thus reducing substrate thickness (or thermal conductivity) would be beneficial. The effect of substrate thickness on $\Delta T_{\max }$ is plotted in figure 5 .

Losses from radiation are less than those from the substrate in device 1 . In device 2 , where a thinner substrate and lower conductivity films are used, radiation is the dominant loss. The performance of each device is plotted as a function of device length in figure $5 \mathrm{~b}$. As the device is made larger the radiation effects begin to dominate.

Losses in the very fine 0.001 inch diameter k-type thermocouple wire used are relatively small. For a 0.003 inch diameter thermocouple, however, the thermal loss will be over 5 times larger since the loss goes as the radius of the thermocouple to the three halves power (equation 8). In this discussion we have considered the thermocouple losses to be spread over the entire cold junction of the device. In practice the thermocouple could cause a local hot spot if it were a more significant heat load.

Finally, depositing thicker films or films with higher ZT would also improve performance. As can be seen in table 1 the n-type $\left(\mathrm{Bi}_{0.95} \mathrm{Sb}_{0.05}\right)_{2}\left(\mathrm{Te}_{0.95} \mathrm{Se}_{0.05}\right)_{3}$ film could be improved considerably before bulk properties are reached. Increasing film thickness diminishes the importance of the parasitic thermal loads associated with thin films which we have investigated in this work. The dramatic improvement in $\Delta T_{\max }$ with increasing film thickness can be seen in figure $5 c$.

\section{Conclusion}

Excellent agreement is found between the values calculated for the device from the measured parameters and those measured experimentally from actual devices (table 1). This agreement suggests that all the relevant thermal loading is accounted for in the model. Using the analytical model developed here will lead to improved device performance.

\section{Acknowledgments}

Funding for this project was provided by ARPA, Larry Dubois, program manager. We would like to acknowledge D. Makowiecki for use of the sputtering chamber. This work was done under the auspices of the U.S. DOE by Lawrence Livermore National Laboratory under Contract No. W-7405-Eng-48.

\section{References}

[1] L.D. Hicks, M.S. Dresselhaus, "Use of Quantum-Well Superlattices to Obtain a High Figure of Merit from Nonconventional Thermoelectric Materials," Appl. Phys. Lett. 63, 3230-3232, 1993.

[2] I.H. Kim, B.G. Min D.H. Lee, "Effects of Thickness and Processing Variables on the Performance of $(\mathrm{Bi}, \mathrm{Sb})_{2}(\mathrm{Te}, \mathrm{Se})_{3}-$ based Film Type Thermoelectric Modules", Proc. of the XIV International Conference on Thermoelectrics, St. Petersburg, Russia, 145-150, 1995.

[3] A.V. Wagner, R.J. Foreman, L.J. Summers, T.W. Barbee, Jr., J.C. Farmer, "Synthesis and Evaluation of Thermoelectric Multilayer Film", to be published in Proc. of the XV Intemational Conference on Themoelectrics, Pasadena, CA, 1996.

[4] A.V. Wagner, R.J. Foreman, L.J. Summers, T.W. Barbee, Jr., J.C. Farmer, "Multilayer Thin Film Thermoelectrics Produced by Sputtering", Proc. of the XIV International Conference on Thermoelectrics, St. Petersburg, Russia, 283-287 1995.

[5] A.V. Wagner, R.J. Foreman, L.J. Summers, T.W. Barbee, Jr., J.C. Farmer, "Multilayer Thermoelectric Films: A Strategy for the Enhancement of ZT," Proc. 30th Intersociety. Energy Conversion Engineering Conference, Orlando, Florida, 3, 87-92, 1995.

[6] H.S. Carslaw, and J.C. Jaeger, "Conduction of Heat In Solids", Oxford University Press, 135, 1959. 